Published by Al-Nahrain College of Medicine P-ISSN 1681-6579

E-ISSN 2224-4719

Email: iraqijms@colmed-alnahrain.edu.iq

http://www.colmed-alnahrain.edu.iq

http://www.iraqijms.net

Iraqi JMS 2019; Vol. 17(1)

\title{
Detection of TTV Antigen in Patients with Hepatitis HBV and HCV
}

\author{
Ealaf A. Khudair ${ }^{1}$ BSC, Arwa M.A. Al-Shuwaikh² PhD, Nawal M. Farhan ${ }^{3}$ CABM, FICMS, GH \& H \\ ${ }^{1}$ Alnahrain Company for Drug Industry, Baghdad, Iraq, ${ }^{2}$ Dept. of Microbiology, College of Medicine, Al-Nahrain \\ University, Baghdad - Iraq, ${ }^{3}$ Gastroenterology and Hepatology Teaching Hospital, Baghdad, Iraq
}

\begin{abstract}
Background Several lines of evidence have suggested the presence of new hepatitis agents, in addition to established hepatitis viruses A-E. Torque Teno virus (TTV) was more prevalent in patients with hepatitis, so it was thought to have hepatotropic properties.

Objective To detect TTV Ag in apparently healthy blood donors and patients infected with chronic hepatitis $B$ virus (HBV) or chronic hepatitis $C$ virus (HCV) by enzyme linked immunosorbent assay (ELISA) technique. Also, to find out any possible association between the study population demographic data and TTV status.

Methods This study was conducted from the beginning of November, 2017 to the end of March, 2018. Serum samples were collected from 50 patients who had chronic hepatitis HBV or HCV and attended to Gastroenterology and Hepatology Teaching Hospital. Also, sera were collected from a total 43 healthy blood donors from the Blood Donation Center in Al-Imamein Al-Kadhimein Medical City. The clinical characteristics of both patients and controls such as alanine transaminase $(A L T)$, aspartate transaminase (AST), hepatitis C virus antibody (HCV-Ab), hepatitis B surface antigen ( $\mathrm{HBsAg}$ ) and hepatitis $B$ core antibody ( $\mathrm{HBcAb})$ were obtained from hospital records. Serum samples were tested by ELISA technique for detection of TTV Ag.

Results TTV was detected in $89.2 \%$ (33 out of 37) of the HBV-positive patients and in $30.8 \%$ (4 out of 13) of the HCV-positive patients versus $23.3 \%$ of the healthy blood donor (10 out of 43 ). Results of this study showed that the prevalence of TTV in HBV patients is significantly higher than HCV patients and healthy blood donors. Concerning risk factors, it was found that there was statistically significant relationship between TTV positivity and mean level of ALT but results indicated that the presence of TTV was not associated with AST, sex, age and history of transfusion, surgery and tattooing.

Conclusion TTV may play a role in hepatitis and its presence was associated with biochemical signs of liver disease, and among patients infected with chronic HBV or HCV.

Keywords Torque Teno virus, hepatitis $B$ virus, hepatitis $C$ virus, healthy blood donors

Citation khudair EA, Al-Shuwaikh AMA, Farhan NM. Detection of TTV antigen in patients with hepatitis HBV and HCV. Iraqi JMS. 2019; 17(1): 43-49. doi: 10.22578/IJMS.17.1.7
\end{abstract}

List of abbreviations: ALT = Alanine transaminase, AST $=$ Aspartate Aminotransferase, TTV= Torque Teno virus

\section{Introduction}

7 orque Teno virus (TTV) is a relatively small $(3.8 \mathrm{~kb})$ circular ssDNA virus that has been classified in the newly characterized family Anelloviridae (1,2). It was suspected of being significantly associated with hepatitis (3).

TTV is characterized by extremely high prevalence, with the relatively high level of genomic heterogeneity. It was detected in the liver and blood of people with hepatic pathologies of unknown etiology. Blood transfusion was initially indicated as the 
principal of viral transmission due to direct contact with contaminated blood. However, there are new routes of transmission that have been recognized, due to the presence of the virus in different body fluids such as in feces, saliva and also in river water contaminated by sewage ${ }^{(4)}$.

Wide ranges of TTV virus infection is reported in individuals with liver disease, Human Immunodeficiency Virus (HIV) positive, intravenous drug users, thalassemic patients and patients on maintenance hemodialysis (5). From preliminary reports, two characteristics of TTV infection have emerged rendering it as a potential cause of liver disease. First, Nishizawa et al. ${ }^{(6)}$ reported the presence of TTV-DNA in the sera of patients with non-A-E hepatitis to reveal a close correlation with alanine transaminase (ALT) level. Second, Okamoto et al. (7) discovered that TTV-DNA levels in liver tissue were equal or one hundred times more than those in serum, suggesting that this virus replicated in the liver. The overall TTV viremia rates in hepatitis $B$ virus (HBV) and hepatitis $C$ virus (HCV) positive subjects were $90.75 \%$ and $84.9 \%$, respectively as well as $81.4 \%$ in healthy individuals in Qatar (3).

In this study, we aimed to determine the frequency of TTV viremia by performing enzyme linked immunosorbent assay (ELISA) in healthy blood donors and patients with chronic HBV or HCV. Also, estimation of demographic data such as age, sex, aspartate aminotransferase (AST) and alanine transaminase (ALT) and history of underlying medical conditions.

\section{Methods \\ Subjects}

This study included a total of 93 blood samples collected from 50 patients with chronic HBV or HCV from the Gastroenterology and Hepatology Teaching Hospital for the period from the beginning November, 2017 to the end of March, 2018. In addition, 43 blood samples were collected from healthy blood donors who attended the Blood Donation Center in AlImamein Al-Kadhimein Medical City. Blood sample was drawn from each patients and control by venipuncture, after obtaining informed consent. The protocol for the research project was approved by the Institutional Review Board (IRB) of College of Medicine, AlNahrain University.

\section{Specimens collection}

Serum samples were collected from all hepatitis patients and healthy blood donors by venipuncture of the median cubital vein. Five (5) $\mathrm{ml}$ blood samples were collected in sterile gel tubes without any anticoagulant, allowed to clot in the room temperature within 1 hour of its collection before centrifugation at 3,000 rpm for 10 minutes, divided into aliquots then immediately stored frozen at $(-44){ }^{\circ} \mathrm{C}$ until be used.

\section{Immunoassay}

Ninety-three samples were tested for qualitative detection of TTV Ag by ELISA kit (Abbexa, England). The wells of the microtiter plate were coated with an antibody specific to TTV Ag. The procedure was done following manufacturer's instructions. For sample preparation, each sample was diluted (1:5) with sample diluent buffer before adding into the set wells by adding $10 \mu \mathrm{l}$ of serum to $50 \mu \mathrm{l}$ sample diluents which supplied with the kit. Blank, positive and negative controls were included in ELISA run. The optical densities (O.D.) of each well were measured at wave length $(450 \mathrm{~nm})$. The calculated absorptions for the patient and control serum were compared with the cut off value. The cut off value $=$ absorbance of negative control +0.15 , if the absorbance of the sample is equal or higher than the cut off value, the test sample is considered positive, otherwise the test sample is considered negative.

\section{Statistical analysis}

Analysis of data was carried out using Statistical Packages for Social Sciences- version 19 (SPSS19). Categorical data presented as count and percentage; the differences were examined by Chi-square test $\left(\chi^{2}\right.$-test). Whereas, numerical data presented as mean, standard deviation and 
evaluated by using independent sample T-test, statistical significance was considered whenever the $P$ value for the test of significance was equal or less than 0.05 .

\section{Results}

This study included fifty hepatitis patients with HBV or HCV infection, with mean age of $36.20 \pm$ 13.4 S.D. year and 43 healthy blood donors that randomly selected persons considered as control group, their mean age was $35.22 \pm 9.8$
S.D. year. Thirteen out of $50(26 \%)$ patients were hepatitis $C$ positive while 37 out of 50 (74\%) patients were hepatitis $B$ positive. Regarding the sex distribution, 24 hepatitis patients and 40 healthy blood donors were males. Females represented 26 and 3 in patients and healthy blood donors, respectively. T-test showed that the mean values of liver function test parameters i.e. ALT and AST were higher in hepatitis patients than in healthy blood donors as shown in Table 1.

Table 1. Characteristics of the subjects

\begin{tabular}{cccc}
\hline Parameters & $\begin{array}{c}\text { HBV or HCV Patients } \\
(\mathbf{n}=\mathbf{5 0})\end{array}$ & $\begin{array}{c}\text { Healthy blood donors } \\
(\mathbf{n}=\mathbf{4 3})\end{array}$ & P. value \\
\hline Age & $36.20 \pm 13.4$ & $35.22 \pm 9.8$ & 0.679 \\
Sex (M/F) & $24 / 26$ & $40 / 3$ & 0.000 \\
ALT (U/I) & $50.9 \pm 49.7$ & $16.0 \pm 6.0$ & 0.000 \\
AST (U/I) & $52.6 \pm 56.5$ & $22.96 \pm 7.7$ & 0.001 \\
\hline
\end{tabular}

TTV was detected in $23.3 \%$ of the healthy blood donor (10 out of 43 ) as well as in $30.8 \%$ of the HCV-positive individuals (4 out of 13) and in $89.2 \%$ of the HBV- positive individuals (33 out of $37)$, the difference was statistically significant $(P<0.05)$ as shown in Table 2.

Results showed that there was a significant difference in sex between TTV positive and negative individuals. TTV infection was found in higher proportions among females than males (74.1\% vs $40.9 \%)(P=0.004)$. Concerning the risk factors for study individuals, there is no significant difference regarding history of blood transfusion, tattooing and surgery in TTV positive and negative individuals $(P>0.05)$, as shown in Table 2.

Results showed that there was no statistically significant difference between TTV positive and TTV negative subjects as regards AST but there was a significant difference between TTV positive and TTV negative as regards ALT as shown in Table 3. The mean age of TTV positive vs. TTV negative was $(34.66 \pm 11.651,36.17 \pm$
11.361, $P>0.05)$, so there was no significant difference in the mean age between TTV positive and negative individuals.

\section{Discussion}

Despite that the pathogenicity of TTV is not fully clear, but undoubtedly TTV can infect patients already infected with other viruses previously (7). Since TTV considered as post-transfusion virus that cause hepatitis, the most important high-risk persons are those infected with HBV and $\mathrm{HCV}{ }^{(8)}$.

Epidemiological studies have shown that TTV is described worldwide in various populations. Many studies have been done trying to assess whether TTV could cause liver diseases $(9,10)$. Although TTV has absence of apparent pathogenicity, the phenomenon of co-infection is common in TTV; it has been detected in coinfection with many other viral species and is known to aggravate various infections like liver disorders, cancer of pancreas, rhinitis and asthma ${ }^{(11)}$. 
Table 2. Detection of TTV Ag and clinical data in studied population (total number $n=93$ )

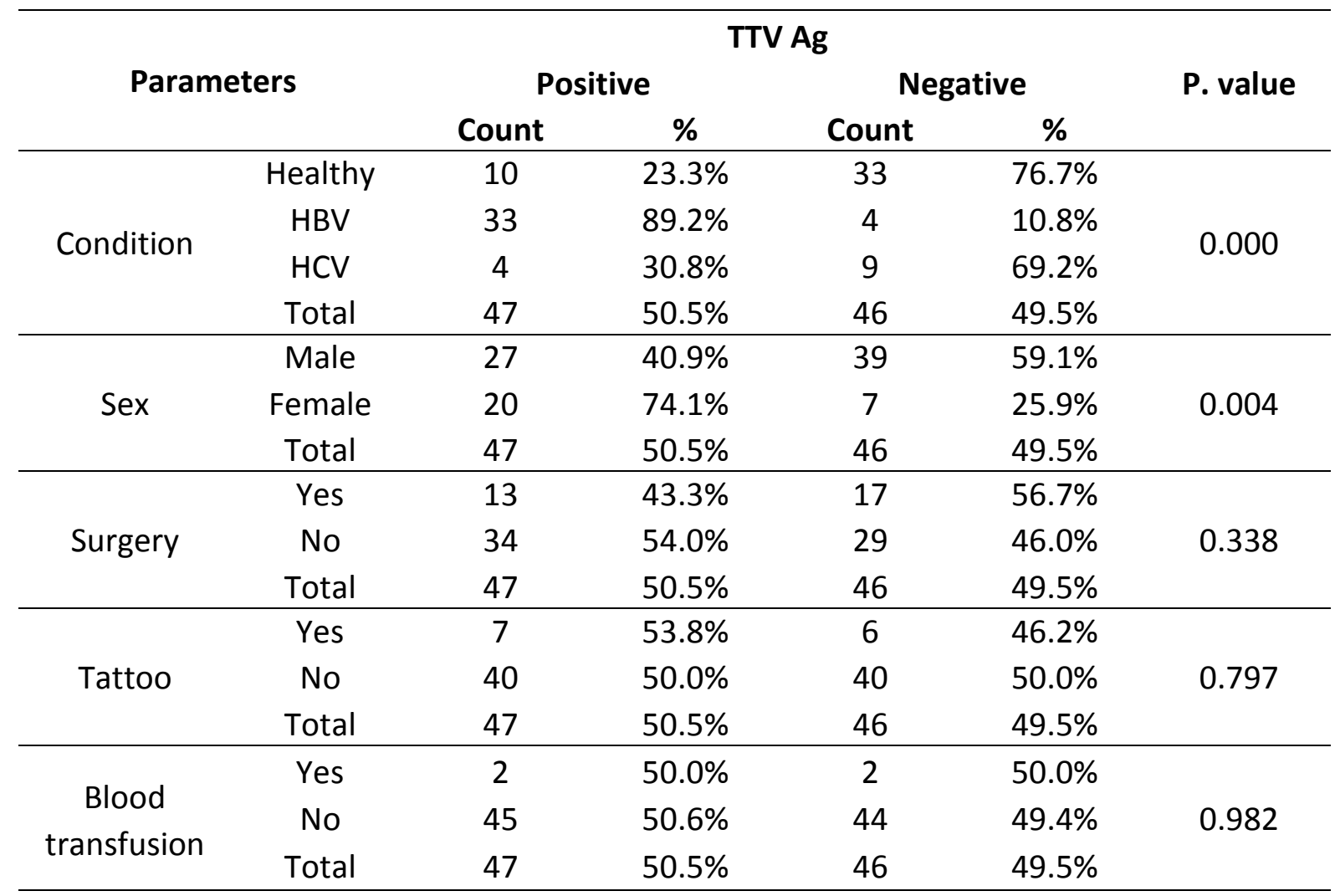

Table 3. Age, serum ALT and AST level (U/L) in relation to TTV Ag status in the studied populations (total number $n=93$ )

\begin{tabular}{cccc}
\hline Parameters & TTV Ag & Mean \pm S.D. & P. value \\
\hline \multirow{2}{*}{ Age (Year) } & Positive & $34.66 \pm 11.651$ & \multirow{2}{*}{0.527} \\
& Negative & $36.17 \pm 11.361$ & \multirow{2}{*}{0.041} \\
\hline \multirow{2}{*}{ ALT(U/I) } & Positive & $44.51 \pm 50.285$ & \multirow{2}{*}{0.352} \\
& Negative & $26.11 \pm 23.545$ & \\
\hline \multirow{2}{*}{ AST (U/I) } & Positive & $45.94 \pm 50.904$ & \\
& Negative & $32.63 \pm 35.063$ & \\
\hline
\end{tabular}

In this study, the overall frequency of TTV infection was 50.5\%, TTV Ag was detected in $23.3 \%$ of the healthy blood donor as well as in $53.8 \%$ of the HCV-positive individuals and in 89.2\% of the HBV- positive individuals as shown in Table 2 which is higher than the previously reported frequency in patients ${ }^{(12)}$. This is may be due to differences in diagnostic techniques, study sample size, and geographic distribution.
On the other hand, these data were in concordance with those reported in patients with chronic hepatitis or cirrhosis in Japan, Taiwan and Iran (13,14).

In comparison with laboratory parameters of liver injury, no significant correlation was observed in the level of AST in healthy blood donors and hepatitis patient with or without TTV infection but there was a significant 
difference between TTV positive and TTV negative as regards ALT as shown in Table 3, suggesting that the presence of TTV could be associated with more severe liver damage in hepatitis patient. This is similar to Nishizawa et al (6) results, who observed that there is an influence of TTV infection on the worsening of liver enzymes in hepatitis patients; hence there is a pathogenic role of TTV in causing liver injury. In contrast, regarding the biochemical parameters another study reported the absence of significant difference in liver enzyme levels between the TTV-positive and negative hepatitis patients ${ }^{(15)}$.

Considering age of studied population most of the TTV positive individuals were with mean age of $34.66 \pm 11.651$ year. However, there was no significant difference in age between TTV positive and TTV negative in healthy blood donors and hepatitis patients. This result found to be consistent with Chattopadhyay et al. (16) results, who reported a statistically insignificant difference between the age of TTV positive and TTV negative infected individuals. One more study reported that there was a significant correlation between TTV infection and age (17). Non-significant association of age in this study could be explained by the fact that this kind of viral infection could occur in people with any age ${ }^{(16)}$.

TTV infection was found in nearly higher proportions among females than males $(74.1 \%$ vs. $40.9 \%)$. The most acceptable explanation for this result is the possibility that women being mostly as multiparous, nosocomial way of transmission as well as the accumulative exposure to various sources (family, occupation, hospital). This is in agreement with another previous study, Al-Qahtani et al. (18), who mentioned that infection rate with TTV was slightly higher in females. In contrast, Chattopadhyay et al. (16) described a notable difference in TTV prevalence according to sex with a higher proportion of males among TTV positive patients.

Considering history of blood transfusion, the present study did not report any significant association with TTV infection, this may be due to the limited number of individuals giving a history of blood transfusion in this study (4 out of 100). In addition, many studies reported that TTV was not associated with blood transfusion history indicating that blood transfusion transmission is not the only way for people to be infected with TTV $(17,19)$. However, another study showed a significant difference between TTV positive and negative patients regarding blood transfusions (17).

The fact that TTV is also observed in healthy blood donors without history of blood transfusion suggests that it could be transmitted by ways other than blood and injection. Possible mechanisms of transmission need consideration include fecal-oral route, saliva, amniotic fluid from TTV-positive women, breast milk, bile and other tissues ${ }^{(7)}$.

This study showed that no significant association was found between TTV positivity and individuals with history of surgery. This is in disagreement with another previous study which suggested nosocomial transmission of the virus, as a consequence of using contaminated fomites and intravascular catheters, as well as with postoperative wounds and elasticized surgical bandages in surgical patients and surgical wound. Also, instruments, equipment, wound dressing, all may act as sources of infection as a result of contamination from blood and blood products $(16,20)$.

Results from epidemiologic studies regarding the risk of viral infections among tattooed individuals are conflicting $(21,22)$, therefore, this study also tried to investigate the relationship between TTV infection and tattoo in order to determine the risk of transmission of TTV infection. The present study didn't find any association between history of tattooing and TTV infection in all study groups $(P>0.05)$, these findings are consistent with a previous study in Japan ${ }^{(17)}$.

To date, solid proof for the association of TTV in chronic liver damage or any involvement with hepatocellular carcinoma, acute sporadic hepatitis, or non-A-E hepatitis has not been confirmed. Another study suggested that these viruses may serve as commensal organisms that have some beneficial role in maintaining homeostasis in the host. These types of "harmless" viruses have been referred to as orphan viruses (23). These viruses had been 
isolated but had not yet been associated with any known disease. So, they considered as "simple guests," although it may be difficult to attribute the term "guest" or "endosymbiont" to viral agents which, due to their biological characteristics, alter normal cell functions ${ }^{(24)}$.

If TTV is a hepatitis agent, it causes illness in only a small minority of infected persons, the difference possibly depending upon immune responsiveness, host susceptibility or viral load. The finding that TTV most likely to be found in serum of an HIV-infected intravenous drug abuser support the possibility that these high viral titers are mainly due to the immunosuppression (17).

This study concluded that the frequency of TTV Ag was significantly higher in HBV patients than $\mathrm{HCV}$ patients and healthy blood donors and may play a role in hepatitis. Detection of TTV among blood donors suggests that these viruses are also transmitted by different routes rather than blood transfusion.

\section{Acknowledgments}

To the staff members in Blood Donation Center in Al-Imamein Al-kadhimein Medical City, Gastroenterology and Hepatology Teaching Hospital for their assistance in samples collection.

\section{Author contribution}

All authors contributed to this manuscript. Dr. Al-Shuwaikh: design, interpreted and arranged this manuscript, khudair: performed all the laboratory work, implementation and progress of this study, Dr. Farhan: helps in clinical aspect and collection of samples.

\section{Conflict of interest}

There is no conflict of interest.

\section{Funding}

This work was partially supported by L'OrealUNESCO for Women in Science Levant and Egypt.

\section{References}

1. Muljono DH, Nishizawa T, Tsuda F, et al. Molecular epidemiology of TT virus (TTV) and characterization of two novel TTV genotypes in Indonesia. Arch Virol. 2001; 146(7): 1249-66.
2. Biagini P. Human circoviruses. Vet Microbiol. 2004; 98(2): 95-101.

3. AbuOdeh $\mathrm{R}$, Al-Mawlawi $\mathrm{N}$, Al-Qahtani $\mathrm{AA}$, et al. Detection and genotyping of Torque Teno virus (TTV) in healthy blood donors and patients infected with HBV or HCV in Qatar. J Med Virol. 2015; 87(7): 118491. doi: 10.1002/jmv.24146.

4. Mazzola JC, Saito PK, Yamakawa RH, et al. Prevalence of Torque Teno virus in healthy donors of Paraná State, southern Brazil. Rev Bras Hematol Hemoter. 2015; 37(5): 336-40. doi: 10.1016/j.bjhh.2015.07.005.

5. De Castro Amarante MF, Kashima S, Covas DT. TT virus (TTV) genotyping in blood donors and multiple transfused patients in Brazil. Virus Genes. 2007; 35(3): 503-9. doi: 10.1007/s11262-007-0124-x.

6. Nishizawa $T$, Okamoto $H$, Konishi $K$, et al. A novel DNA virus (TTV) associated with elevated transaminase levels in posttransfusion hepatitis of unknown etiology. Biochem Biophys Res Commun. 1997;241(1):92-7. doi: 10.1006/bbrc.1997.7765.

7. Okamoto $\mathrm{H}$, Nishizawa $\mathrm{T}$, Kato $\mathrm{N}$, et al. Molecular cloning and characterization of a novel DNA virus (TTV) associated with posttransfusion hepatitis of unknown etiology. Hepatol Res. 1998; 10(1): 1-16. doi: https://doi.org/10.1016/S1386-6346(97)00123-X.

8. Gerner P, Wirth S. [Characteristics and clinical significance of new hepatotropic virus's hepatitis $G$ virus, TT-virus and SEN-virus]. Monatsschrift Kinderheilkd. 2002; 150(1): 19-26. https://doi.org/10.1007/s112-002-8175-3.

9. Tanaka Y, Primi D, Wang RY, et al. Genomic and molecular evolutionary analysis of a newly identified infectious agent (SEN virus) and its relationship to the TT virus family. J Infect Dis. 2001; 183(3): 359-67. doi: 10.1086/318091.

10. Hino S, Miyata H. Torque Teno virus (TTV): current status. Rev Med Virol. 2007; 17(1): 45-57. doi: 10.1002/rmv.524.

11. Tyschik EA, Shcherbakova SM, Ibragimov RR, et al. Transplacental transmission of Torque Teno virus. Virol J. 2017; 14(1): 92. doi: 10.1186/s12985-0170762-0.

12. Jahan S, Rizvi M, Azam M, et al. SEN and Torque Teno virus: Putative agents of Non-A-E viral hepatitis. J Immunol Vaccine Technol. 2015; 1(1): 104.

13. Wolff C, Diekmann A, Boomgaarden M, et al. Viremia and excretion of TT virus in immunosuppressed heart transplant recipients and in immunocompetent individuals. Transplantation. 2000; 69(3): 351-6.

14. Pirouzi A, Bahmani M, Feizabadi MM, et al. Molecular characterization of Torque Teno virus and SEN virus co-infection with HIV in patients from Southern Iran. Rev Soc Bras Med Trop. 2014; 47(3): 275-9. doi: http://dx.doi.org/10.1590/0037-8682-0073-2014.

15. Maggi $F$, Pistello $M$, Vatteroni $M$, et al. Dynamics of persistent TT virus infection, as determined in patients treated with alpha interferon for concomitant hepatitis C virus infection. J Virol. 2001; 75(24): 11999-2004. doi: 10.1128/JVI.75.24.1199912004.2001. 
16. Chattopadhyay S, Das BC, Gupta RK, et al. Presence of TT virus infection in chronic hepatitis patients from a hospital in New Delhi, India. Indian J Med Res. 2005; 122(1): 29-33.

17.Zandieh T, Babaahmadi B, Pourfathollah A, et al. Transfusion transmitted virus (TTV) infection in thalassemic patients. Iran J Public Health. 2005; 34(4): 24-9.

18. Al-Qahtani AA, Alabsi ES, AbuOdeh R, et al. Prevalence of anelloviruses (TTV, TTMDV, and TTMV) in healthy blood donors and in patients infected with $\mathrm{HBV}$ or HCV in Qatar. Virol J. 2016; 13(1): 208. doi: 10.1186/s12985-016-0664-6.

19. Ukita $M$, Okamoto $H$, Nishizawa $T$, et al. The entire nucleotide sequences of two distinct TT virus (TTV) isolates (TJN01 and TJN02) remotely related to the original TTV isolates. Arch Virol. 2000; 145(8): 154359. doi: 10.1007/s007050070075.

20. Umemura T, Alter HJ, Tanaka E, et al. Association between SEN virus infection and hepatitis $C$ in Japan. J Infect Dis. 2001; 184(10): 1246-51. doi: 10.1086/324210.

21. Jafari $S$, Buxton JA, Afshar $K$, et al. Tattooing and risk of hepatitis B: A systematic review and meta-analysis. Can J Public Heal. 2012; 103(3): 207-12.
22. Deschesnes $M$, Demers $S$, Finès $P$. Prevalence and characteristics of body piercing and tattooing among high school students. Can J Public Heal. 2006; 97(4): 325-9.

23. Lemon SM, Layden TJ, Seeff L, et al. The 20th United States-Japan Joint Hepatitis Panel Meeting. Hepatology. 2000; 31(3): 800-6. doi: 10.1002/hep.510310338

24. Mushahwar IK. Recently discovered blood-borne viruses: Are they hepatitis viruses or merely endosymbionts? J Med Virol. 2000; 62(4): 399-404.

Correspondence to Dr. Arwa M. A. Al-Shuwaikh E-mail: arwa.mujahid@colmedalnahrain.edu.iq, arwa_alshwaikh_2004@yahoo.com Received Sep. $9^{\text {th }} 2018$ Accepted Dec. $4^{\text {th }} 2018$ 